\title{
Lateral Knee Pain Favors the Diagnosis of Anterior Cruciate Ligament Injury and its Postoperative Assessment Potentially Contributes in Treatment Follow- Up; A Pilot Study
}

\section{Masoome Pourmokhtari}

Jahrom University of Medical Sciences

\section{Ali Razeghi}

Jahrom University of Medical Sciences

Nikta Taghipour ( $\sim$ Niktataghipoor114@gmail.com )

Jahrom University of Medical Sciences

\section{Research Article}

Keywords: lateral knee pain, anterior cruciate ligament tear, anterior cruciate ligament reconstruction, surgery

Posted Date: August 19th, 2021

DOI: https://doi.org/10.21203/rs.3.rs-821068/v1

License: (c) (i) This work is licensed under a Creative Commons Attribution 4.0 International License. Read Full License 


\section{Abstract}

Background: Anterior Cruciate Ligament (ACL) reconstruction is a standard technique for treatment of patients with an $\mathrm{ACL}$ tear. According to clinical observations, lateral knee pain is highly associated with a teared $\mathrm{ACL}$, this correlation so far has remained unnoticed in the literature. In this study, we aim to investigate the relationship of lateral knee pain with diagnosis of torn ACL.

Methods: This pilot study was conducted on 30 candidates of ACL reconstruction. The participants underwent surgical reconstruction of ACL; Then Visual Analogue Scale (VAS) was used to measure pain at three time points of preoperative, first and second postoperative visits.

Results: A total of 26 participants ( $86.7 \%)$ were male with a mean age of $27.3 \pm 5.06$ years. The results demonstrated that the intensity of lateral knee pain felt by the subjects significantly diminished during subsequent postoperative referrals ( $P$-value $<0.001)$. However, other variables including sex, age, and type of surgery (bone-patella tendon-bone and hamstring tendon) caused no significant difference in terms of pain reduction ( $p>0.05)$.

Conclusions: Lateral knee pain is likely related with anterior cruciate ligament tear and favors its diagnosis. Also, the evaluation of lateral knee pain may contribute in Anterior Cruciate Ligament reconstruction (ACLR) monitoring in the follow-up stage.

\section{Background}

Anterior cruciate ligament ( $\mathrm{ACL}$ ) tear is one of the most common injuries of knee cruciate ligaments due to its structure and unique anatomical function in stabilization of the knee joint [1]. The incidence rate of ACL injuries is annually 68.6 per 100,000 people, and it commonly occurs in young individuals who perform strenuous physical activity, and may eventually lead to a chronic instability [2]. According to epidemiological statistics, around 200,000 cases per year are reported in the USA [3]. This injury influences the knee function and causes pain [4], joint instability [5], injury of joint articulation [6], osteoarthritis [7], reduced activity [8], and decreases the quality of the patient's life [9].

Arthroscopic ACL reconstruction (ACLR) is considered as a standard surgical treatment for patients with functional instability of the knee. Two common autografts used in this surgical procedure are bone-patella tendon-bone (BPTB) and hamstring tendon $(\mathrm{HT})$, although which autograft takes precedence over another in terms of being selected as the preferred one is still controversial [10-13].

Nevertheless, some studies have considered BPTB graft as a preferred technique with a high graft stability, especially in young athletes $[14,15]$. On the other hand, other studies have demonstrated that complications like anterior knee pain and kneeling pain are associated with the use of BPTB graft [16]. Although knee pain manifests mainly in all aspects of the anterior, medial, and lateral side of the knee [17], the role of anterior and medial knee pain in the diagnosis of $A C L$ is suspected. The underlying reason could be the fact that anterior knee pain and kneeling pain may develop during 1 to 2 years after reconstruction of ACL [18]. Further, there are several common causes for lateral knee pain mentioned as follows: iliotibial band syndrome, sprains of anterior cruciate ligament, injury of lateral meniscus, patellofemoral pain syndrome (PFPS), osteoarthritis (OA), biceps femoris tendinopathy or tendonitis, synovitis, and sprain of superior (proximal) tibiofibular joint [19-22]. Nonetheless, lateral knee pain has been commonly associated with anterior cruciate ligament tear based on our clinical observations, it is pertinent to point out that this association has remained so far unnoticed in the literature. Thus, the present study majorly aims to 
evaluate the correlation of lateral knee pain with a torn $\mathrm{ACL}$, and investigates the impact of demographic characteristics and type of autografts on the intensity of lateral knee pain.

\section{Methods}

\section{Design and target population}

This pilot study was conducted on 30 candidates of surgical ACLR who referred to Peymanieh Hospital (affiliated to Jahrom University of Medical Sciences) between December 2018 to December 2019. The inclusion criteria upon the visit were as follow: willingness to participate in the study, MRI confirmation of a complete ACL rupture before the operation, absence of any chronic disease or cancer history, absence of ankle fracture, osteoarthritis in knee and infection in knee. On the other hand, unwillingness and uncooperativeness to resume participation, partial ACL tear, and absence or loss of eligibility requirements during the study were considered as exclusion criteria for the subjects. Also, a written consent was obtained from the patients prior to admission in the study. In order to monitor the patient's post-operatively, they were followed-up in two postsurgical visits, once at the end of the second week and another at the end of sixth week after surgery.

\section{Study population and sampling method}

The sample size was determined according to Khorpong and Kovindha's study (2018) [12]. Using Altman's nomogram, with a given standard difference $=0.78$, estimation of $95 \%$ confidence interval, power of $80 \%$ and attrition rate of $15 \%$, a sample size of 30 individuals was yielded (Gpower program was utilized for power analysis and sample size calculation). The patients were selected from the candidate individuals of ACLR who referred to Peymanieh Hospital (affiliated to Jahrom University of Medical Sciences) through the method of convenient sampling. The candidates were admitted to the study given a complete ACL rupture was evident in their magnetic resonance imaging.

\section{Intervention}

Our patients underwent ACLR either with BPTB autograft or HT autograft, and followed the same rehabilitation protocol as that meant for the strengthening of the hamstring or the quadriceps muscle. The rehabilitation was offered with goals and that have been similarly defined in the pre-rehabilitation stages for both patient groups. The anterior cruciate ligaments were reconstructed by an experienced surgeon and his team. Except for autograft harvest and fixation method of the leg bone, a similar surgical technique was used for all patients for performing the rest of the interventions.

During the operation, patients were given a reginal spinal anesthesia. First, betadine was used to prepare and to stain the surgical knee field, then examination was performed and ACL tear was confirmed by the aid of arthroscopy. Afterwards, meniscal cartilage injuries were evaluated, and if deemed necessary were repaired. In surgical repair by BPTB, the graft was taken from the central third of patella tendon comprising $2.5 \mathrm{~cm}$ of bony attachments of both patella and tibial tuberosity through a $4 \mathrm{~cm}$ longitudinal incision over the patellar tendon. Subsequently, the patellar tendon was repaired by using an interrupted suture pattern in the posterior half with the paratenon and was then closed with an absorbable suture.

In patients with surgical reconstruction using $\mathrm{HT}$, the tendons of semitendinosus or gracilis were harvested from a 3 $\mathrm{cm}$ surgical incision at the level of the tibial tubercle. Following the graft harvest, drilling of femoral tunnel was performed through the antero-medial portal with an appropriate reamer guide according to the graft diameter. To 
determine the site of tunnel placement, ACL stump (footprint), intercondylar notch, lateral and medial epicondyles were considered as the anatomical landmarks.

For the previous ACL stump, a tibial tunnel was drilled and the anterior horn of lateral meniscus and tibial spine were considered as the anatomical landmarks, with entrance being the tunnel on medial side of tibia and exit site being the tibial plateau.

Further, for the reconstruction of the ACL stump, a tunnel was drilled in the tibia, from the medial aspect of the tibia to the right side of the tibial plateau, while taking into account the anterior horn of lateral meniscus and the tibial spine as the anatomical landmarks.

Finally, the utilized grafts were fixated with a cortical suspensory button in the patients of HT graft group and with a bioabsorbable interference screw in the patients of BPTB graft group. No drain was postoperatively inserted in any patient. On the second postoperative day, the patients were allowed to stand up with two walking crutches and standing braces, and were encouraged to walk as the braces was locked in full extension. All the patients were recommended for postsurgical physiotherapy and rehabilitation in order to improve the functional ability of muscles surrounding the knee.

\section{Data collection}

The tool employed to collect the data was a questionnaire composed of two parts: I) demographic information including age, gender, and mechanism of knee injury, II) the Visual Analogue Scale (VAS) to measure the pain intensity in patients. VAS is a measurement instrument using a 10-cm-scaled line continuum where scale 10 indicates "unbearable pain" and 0 represents "no pain", whereby the patient marks the severity of pain on a horizontal line. Obtaining a score range of 1-3 describes mild pain, 4-7 moderate pain, and 8-10 severe pain. The reliability and stability of VAS have been internationally accredited by many studies, and the reliability of this scale in Iran has been confirmed with a given correlation coefficient of $r=0.88$ [23-25].

\section{Statistical analysis}

Data analysis was performed by descriptive and inferential statistics through repeated measures ANOVA model using SPSS version 21. To compare the changes within the groups, Chi-square test and T-test were employed. A statistical significance was considered when $\mathrm{P}<0.05$.

\section{Ethical considerations}

The procedures, purposes, and importance of the research were explained to the participants and a written consent was taken from them before their admittance to the study. In all the phases of the study, the researchers adhered to the Declaration of Helsinki (set of ethical principles regarding human experimentation developed for the medical community) and maintained patient privacy and confidentiality. All the study expenses were financed by the researchers and none of the patients were charged for any additional costs. This research was commenced on getting an approval by the Research \& Technology Deputy of Jahrom University of Medical Sciences, and was also ethically approved by this University's Ethics Committee (Application ID: IR.JUMS.REC.1397.095).

\section{Results}

Among the participants, $86.7 \%$ (26 patients) of the patients were male. The mean age of participants was $27.3 \pm 5.06$ years, ranging from 17 as the youngest to 43 as the oldest. The knee injury in the majority of the patients (18 subjects, $60 \%$ ) had been caused by playing soccer (Table 1). 
Table 1

Demographic features of patients with ACL injury

\begin{tabular}{|llll|}
\hline Variables & Groups & Number of patients & Percentage \\
\hline Sex & & & \\
\hline & Male & 26 & 86.7 \\
\hline Female & 4 & 13.3 \\
\hline Age group (years) & 12 & \\
\hline$\leq 25$ & 13 & 40 \\
\hline $26-30$ & 5 & 43.3 \\
\hline$>30$ & & 16.7 \\
\hline Mechanism of knee injury & 21 & \\
\hline Sport accident & 4 & 70.9 \\
\hline Motor vehicle accident & 3 & 13.3 \\
\hline Falling down & 2 & 10 \\
\hline Others & & 6.6 \\
\hline Bype of surgery & 16 & 53.3 \\
\hline Bone-Patellar tendon-bone & 14 & 46.7 \\
\hline Hamstringtendon &
\end{tabular}

The comparison of knee pain intensity in the lateral aspect was made before and after surgery at three different time points (Table 2). In preoperative visit, $66 \%$ and $33 \%$ of the patients experienced severe (8-10) and moderate (4-7) pain intensity, respectively. At the end of second postoperative week (first postoperative visit), the number of patients experiencing moderate pain increased $(40 \%)$, whereas the number of patients with severe pain decreased $(30 \%)$. Also, more patients were categorized in mild pain continuum (0-3) in first post-operative visit compared to that of the preoperative visit. After a month, only a few patients (3.3\%) complained about severe lateral knee pain at the end of sixth week or second postoperative visit and majority of the patients reported a score range of $0-3$ for their lateral knee pain. To compare the mean pain intensity reported by patients at three stages of pre-operative visit, first postoperative visit, and second postoperative visit, a repeated measures ANOVA model was used. Similar to patient distribution, a reduction in mean intensity of lateral knee pain was noticed. Through the analysis of repeated measure ANOVA, the pain intensity reduced with a noticeable descending trend from $6.56 \pm 2.68$ to $3.33 \pm 2.63$ in the consecutive visits $(P<0.001)$ (Table 2). Also, post hoc multiple comparison compensated with Bonferroni correction revealed that mean values of pain intensity in these three points were pairwise significantly different $(P<0.05)$. 
Table 2

Frequency distribution and mean intensity of lateral knee pain at preoperative and postoperative visits measured by VAS

\begin{tabular}{|c|c|c|c|c|c|c|c|c|c|c|c|c|c|}
\hline Pain intensity & 0 & 1 & 2 & 3 & 4 & 5 & 6 & 7 & 8 & 9 & 10 & $\begin{array}{l}\text { Pain } \\
\text { Intensity } \\
\text { (mean } \pm \\
\text { SD) }\end{array}$ & P.value \\
\hline $\begin{array}{l}\text { At pre- } \\
\text { operative } \\
\text { visit }\end{array}$ & 0 & 6.7 & 3.3 & 6.7 & 3.3 & 16.7 & 3.3 & 13.3 & 16.7 & 20 & 10 & $\begin{array}{l}6.56 \pm \\
2.68\end{array}$ & $\begin{array}{l}< \\
0.001\end{array}$ \\
\hline $\begin{array}{l}\text { At first } \\
\text { postoperative } \\
\text { visit }\end{array}$ & 3.3 & 10 & 6.7 & 10 & 10 & 6.7 & 10 & 13.3 & 10 & 20 & 0 & $\begin{array}{l}5.40 \pm \\
2.90\end{array}$ & \\
\hline $\begin{array}{l}\text { At second } \\
\text { postoperative } \\
\text { visit }\end{array}$ & 23.3 & 10 & 6.7 & 10 & 13.3 & 13.3 & 6.7 & 13.3 & 3.3 & 0 & 0 & $\begin{array}{l}3.33 \pm \\
2.63\end{array}$ & \\
\hline
\end{tabular}

The patients were further categorized based on gender, age and type of surgery to determine if pain intensity measures differed significantly across the groups (Table 3 ). The results obtained from repeated-measures ANOVA concerning the patients' gender indicated that the difference of pain intensity across the visits is significant in both male and female groups, and subsequently, the intensity of the pain lessened $(P<0.001)$. However, no association was found between the intensity of lateral knee pain and sex $(P=0.535)$. Also, the comparison made between pain intensity felt by men and women did not differ significantly from one another $(P=0.835)$. Regarding the reduction of pain intensity in different age groups, it was evident that the difference between pain intensity sensed by patients of different age groups at the preoperative and the postoperative visits was statistically significant and followed a declining trend $(p<0.001)$. However, the pain intensity was not mutually associated with different age groups, i.e., patients followed a similar trend of pain reduction regardless of their age $(P=0.271)$. Concerning the association of gender with pain intensity, the male and female patients of each age group did not differ significantly from each other $(P=0.780)$. Moreover, the patients of both BPTB and HT grafts showed no significant difference in terms of pain intensity with respect to the type of bone graft they had received $(P=0.069)$ (Table 3$)$.

Table 3

Mean intensity of lateral knee pain before and after ACL reconstruction across the groups of sex, age and surgery type

\begin{tabular}{|lllll|}
\hline Variables & \multicolumn{4}{c|}{ Pain Intensity (mean \pm SD) } \\
\cline { 2 - 5 } & & Second postoperative visit & First postoperative visit & Preoperative visit \\
\hline Sex & Male & $7.5 \pm 3$ & $5.25 \pm 3.3$ & $3.25 \pm 3.3$ \\
\cline { 2 - 5 } Age group & Female & $6.42 \pm 2.67$ & $5.42 \pm 2.9$ & $3.34 \pm 2.59$ \\
& Age $\leq 25$ & $5.58 \pm 3.14$ & $5.08 \pm 3.39$ & $3.5 \pm 2.74$ \\
\cline { 2 - 5 } & $25-30$ & $7.30 \pm 1.97$ & $5.76 \pm 2.48$ & $3.07 \pm 2.43$ \\
\hline Type of surgery & BgT $>30$ & $7 \pm 2.91$ & $5.2 \pm 3.11$ & $3.6 \pm 3.36$ \\
\cline { 2 - 5 } & HT graft & $7.71 \pm 2.33$ & $4 \pm 2.5$ & $2.87 \pm 2.21$ \\
\cline { 2 - 5 } & & & $7.56 \pm 2.63$ & $3.85 \pm 3.03$ \\
\hline
\end{tabular}


Regarding the association of autograft harvest method with pain intensity (Fig. 1), the pain intensity reduced at subsequent visits regardless of the surgery type $(P<0.001)$. No relationship was observed between pain intensity and type of surgery; in other words, the pain intensity of patients of both groups of BPTB graft and HT graft followed a similar trend of pain reduction $(\mathrm{P}=0.077)$. Numerically, the pain intensity in patients of HT group was higher compared to that of patients in BPTB group; however, Table 3 clearly demonstrates that this difference is not significant.

\section{Discussion}

According to our clinical observations, it is very likely to find MRI evidences of an ACL injury when patients refer with chief complaint of knee giving way and lateral knee pain. Thus, the presence of pain localized at the lateral aspect of knee usually hints us to consider ACL injury among other differential diagnoses. There are various causes for lateral knee pain which are mainly associated with an injury including iliotibial band syndrome, lateral ligament sprain, lateral cartilage meniscus injuries, PFPS, OA, biceps femoris tendinopathy or tendonitis, synovitis, and sprain of superior (proximal) tibiofibular joint. Although manifestation of lateral knee pain after ACL reconstruction has been addressed in several studies [12,26-29], the association between concurrence of lateral knee pain and ACL tear has not been noted in the literature yet. The data documented by VAS in the present study suggests that the severity of lateral knee pain significantly reduced in consecutive visits of pre- and post-operative periods. Also, the results obtained revealed that the percentage of the patients with severe pain decreased from $46.7 \%$ at the first preoperative visit to $3.3 \%$ at second postoperative visit; while as the percentage of patients with mild pain increased. These findings support the hypothesis of the correlation between lateral knee pain and ACL rupture. The number of studies investigating the lateral knee pain as their main concern is very limited. Kawaguchi et al. [30] noticed lateral knee pain in another aspect; They reported three cases complaining lateral knee pain during rehabilitation exercise post to their ACL reconstruction, and assumed that the lateral knee pain developed due to irritation between Tight Rope (RT) bottom and the autografts bundle used in ACL repair. The lateral knee pain in their cases only occurred when Kawaguchi et al. had surgically removed the RT bottom. Also, the case report of Anandkumar et al. (2018) described a 45-year-old Canadian female with lateral knee pain over the right proximal tibiofibular joint (PTFJ) managed unsuccessfully with rest, medications, bracing, injection, and physiotherapy. Finally, she was clinically diagnosed with PTFJ hypomobility [21]. Also, they reported that the patient was discharged after four treatment sessions of Mulligan's mobilization with movement and taping over the right PTFJ, and finally the patient was pain free and fully functional at the 6th month of her follow-up. However, the case reports of both Kawaguchi et al. [30] and Anandkumar et al. [21] do not assist us to provide any additional evidences to support the idea of the correlation between lateral knee pain and ACL injury, but a relatively rapid disappearance of lateral knee pain in response to a successful treatment poses the potential use of lateral knee pain in ACLR follow-up.

Additionally, the current study also investigated the probable influence of sex, age and type of harvested graft on lateral knee pain intensity. Regarding the type of autografts use, the difference of pain intensity in patients of both BPTB and HT groups was not significant but with a borderline p-value of 0.077 . Since a limited number of patients were investigated in our study, the preference of the two methods of ACL reconstruction in terms of lateral knee pain requires more attention and in-depth studies. In contrast, Okoroha et al. (2016) reported that a significant increase in acute postoperative pain was found when ACL reconstruction was performed with BPTB compared to reconstruction with HS. This cohort study also discovered that patients treated with BPTB were more likely to contact their physician due to pain, and more likely to have a breakthrough pain and low satisfaction with their pain management [31]. Moreover, in a prospective study with a 10-year follow-up, Pinczewski et al. (2007) reported that the number of patients with contralateral ACL re-rupture in the PT group (20) was significantly higher compared to that of HT group 
(9) $(P=0.02)$. Also, more patients reported pain with strenuous activities in PT knees than in HT knees $(P=0.05)$.

Finally, these scientists concluded that excellent results could be obtained with both HT and PT autografts, but their final recommendation was in favor of 4-strand $\mathrm{HT}$ reconstruction to patients due to diminished harvest-site symptoms and radiographic osteoarthritis [32]. Like Pinczewski's study, a long term follow-up was not conducted in our pilot study. However, the relative precedence of PT graft over HT graft in Pinczewski's study did not conform to that of our study.

The pain of other aspects of the knee is also addressed in the literature, particularly the association between anterior knee pain and ACL reconstruction. In this regard, Kovindha et al. (2019) noticed that both anterior knee pain and numbness improved over time (3 to 6 months post-operation) [12]. Likewise, Pinczewski et al. (2007) reported that $33 \%$ of patients suffered from kneeling pain during a 2-year follow-up, and percentage of the patients experiencing kneeling pain during a 10-year follow-up increased to 59\% [32]. The causes of the discrepant results of these two studies with respect to the recovery time of anterior knee pain or incidence rate of kneeling pain are not our concern now, but a more general inference may negate the role of anterior knee pain and kneeling pain in the diagnosis of ACL tear, since these two often appear after ACLR. In addition, the need of a long-term follow-up for the evaluation of other knee pains except lateral knee pain makes us doubt about the assistance of anterior knee pain as well as kneeling pain in the assessment of the success of ACLR. Overall, the paucity of the literature regarding the correlation between lateral knee pain ACL rupture constrained us to find a sound basis for a comprehensive comparison between the studies. Nevertheless, the results of the present pilot may persuade the clinicians to consider the association between lateral knee pain and ACLR as well as efficacy of its operative treatment.

\section{limitation}

The limitations of the current study include a small sample size, discrepancies in rehabilitation and restorative cares maintained by patients, and the difference in rehabilitation equipment used by our patients. Therefore, conduction of further studies with a larger sample size, a randomized sampling from several hospitals, equal numbers of participants from both genders, and similar surgical method with respect to fixation and graft is recommended. Also, evaluation of the etiology of lateral knee pain in the general population can give a better understanding of the specificity of this symptom in diagnosing ACL injuries.

\section{Conclusion}

The present study revealed that the extent of lateral knee pain in candidates of ACL reconstruction at consecutive postoperative visits was significantly reduced and other variables including sex, age, and type of surgery rendered no significant difference with respect to reduction of pain intensity. Also, it can be inferred that lateral knee pain correlates well with a torn ACL. Therefore, lateral knee pain favors ACL tear among other differential diagnoses and it may contribute in treatment follow-up of the patient who underwent an ACL reconstruction.

\section{Abbreviations}

ACL: Anterior cruciate ligament; ACLR : ACL reconstruction; BPTB: bone-patella tendon-bone; HT: hamstring tendon; PFPS: patellofemoral pain syndrome; OA: osteoarthritis; VAS: Visual Analogue Scale; RT: Tight Rope; PTFJ: proximal tibiofibular joint .

\section{Declarations}




\section{Ethics approval and consent to participate}

Written inform consent was obtained from the patients in our study. The purpose of this research was completely explained to the patient and was assured that their information will be kept confidential by the researcher. This research was approved by the ethical committee of Jahrom University of Medical Sciences issued with code No.: IR.JUMS.REC.1397.095.

\section{Consent for publication}

Consent was obtained from the patients regarding the publication of this study

\section{Availability of data and materials}

SPSS data of the participant can be requested from the authors. Please write to the corresponding author if you are interested in such data.

\section{Competing interests}

The authors declare that they have no competing interests.

\section{Funding}

No financial support was received for this study.

\section{Authors' contributions}

MP designed the study while AR collected the data and NT drafted the manuscript. All authors proofread and apporved the final version of the manuscript.

\section{Acknowledgments}

The authors of the study would like to express sincere gratitude to the Clinica/ Research Development Unit of Peymanieh Hospital affiliated to Jahrom University of Medical Sciences for contribution in providing the required services and the participants who participated in the study.

\section{Authors' information}

${ }^{1}$ Department of Orthopedic Surgery, Jahrom University of Medical Sciences, Jahrom, Iran

${ }^{2}$ Student Research Committee, Jahrom University of Medical Sciences,Jahrom, Iran 


\section{References}

1. Mehl J, Diermeier T, Herbst E, et al. Evidence-based concepts for prevention of knee and ACL injuries. 2017 guidelines of the ligament committee of the German Knee Society (DKG). Arch Orthop Trauma Surg. 2018;138(1):51-61.

2. Sanders TL, Maradit Kremers H, Bryan AJ, et al. Incidence of anterior cruciate ligament tears and reconstruction: a 21-year population-based study. Am J Sports Med. 2016;44(6):1502-1507.

3. Xiao W-F, Yang T, Cui Y, et al. Risk factors for noncontact anterior cruciate ligament injury: Analysis of parameters in proximal tibia using anteroposterior radiography. J Int Med Res. 2016;44(1):157-163.

4. Shea KG, Carey JL. Management of anterior cruciate ligament injuries: evidence-based guideline. J Am Acad Orthop Sur. 2015;23(5):e1-e5.

5. van Yperen DT, Reijman M, van Es EM, et al. Twenty-year follow-up study comparing operative versus nonoperative treatment of anterior cruciate ligament ruptures in high-level athletes. Am J Sports Med. 2018;46(5):1129-1136.

6. Arundale AJ, Bizzini M, Giordano A, et al. Exercise-Based Knee and Anterior Cruciate Ligament Injury Prevention: Clinical Practice Guidelines Linked to the International Classification of Functioning, Disability and Health From the Academy of OrthopaedicPhysical Therapy and the American Academy of Sports Physical Therapy. J Orthop Sports Phys Ther. 2018;48(9):A1-A42.

7. Li K, Du J, Huang L-X,et al. The diagnostic accuracy of magnetic resonance imaging for anteriorcruciate ligament injury in comparison to arthroscopy: a meta-analysis. Sci Rep. 2017;7(1):7583.

8. Alanazi HO, Abdullah AH, Qureshi KN. A critical review for developing accurate and dynamic predictive models using machine learning methods inmedicine and health care. J Med Syst. 2017;41(4):69.

9. Erickson BJ, Korfiatis P, Akkus Z, Kline TL. Machine learning for medical imaging. Radiographics. 2017;37(2):505-515.

10. Chee MY, Chen Y, Pearce CJ, et al. Outcome of patellar tendon versus 4-strand hamstring tendon autografts for anterior cruciate ligament reconstruction: a systematic review and meta-analysis of prospective randomized trials. Arthroscopy. 2017;33(2):450-463.

11. Xie X, Liu X, Chen Z, et al. A meta-analysis of bone-patellar tendon-bone autograft versus four-strand hamstring tendon autograft for anterior cruciate ligament reconstruction. Knee. 2015;22(2):100-110.

12. Kovindha K, Ganokroj P, Lertwanich P, Vanadurongwan B. Quantifying anterior knee pain during specific activities after using the bone-patellar tendon-bone graft for arthroscopic anterior cruciate ligament reconstruction. Asia Pac J Sports Med Arthrosc Rehabil Techno. 2019; 15:6-12.

13. Mascarenhas R, Erickson BJ, Sayegh ET, et al. Is there a higher failure rate of allografts compared with autografts in anterior cruciate ligament reconstruction: asystematic review of overlapping meta-analyses. Arthroscopy. 2015;31(2):364-372.

14. Erickson BJ, Harris JD, Fillingham YA, et al. Anterior cruciate ligament reconstruction practice patterns by NFL and NCAA football team physicians. Arthroscopy2014;30(6):731-738.

15. Walters BL, Porter DA, Hobart SJ, et al. Effect of intraoperative platelet-rich plasma treatment on postoperative donor site knee pain in patellar tendon autograft anterior cruciate ligament reconstruction: a double-blind randomized controlled trial. Am J Sports Med. 2018;46(8):1827-1835.

16. Schuette HB, Kraeutler MJ, Houck DA, McCarty EC. Bone-patellar tendon-bone versus hamstring tendon autografts for primary anterior cruciate ligament reconstruction: a systematic review of overlapping meta- 
analyses. Orthop. J. Sports Med. 2017;5(11):2325967117736484.

17. English S, Perret D. Posterior knee pain. Curr Rev Musculoskelet Med. 2010 Oct 1;3(1-4):3-10.

18. Culvenor AG, Collins NJ, Vicenzino B, et al. Predictors and effects of patellofemoral pain following hamstringtendon ACL reconstruction. J Sci Med Sport. 2016;19(7):518-523.

19. Baker RL, Fredericson M. Iliotibial band syndrome in runners: biomechanical implications and exercise interventions. Phys Med Rehabil Clin N Am. 2016;27(1):53-77.

20. Chan W, Chase HE, Cahir JG, Walton NP. Calcific tendinitis of biceps femoris: an unusual site and cause for lateral knee pain. BMJ Case Rep. 2016;2016: bcr2016215745.

21. Anandkumar S, Miller J, J. Werstine R, Young S. Effect of mobilization with movement on lateral knee pain due to proximal tibiofibular joint hypomobility. Physiother Theory Pract. 2018;34(10):813-820.

22. Oda S, Izumi M, Aso K, Ikeuchi M. Impact of medial versus lateral knee pain on deep tissue hyperalgesia and muscle strength. Eur J Pain. 2018;22(10):1757-1766.

23. Rezvani Amin M, Siratinayer M, Ebadi A, Moradian T. Correlation between visual analogue scale and short form of McGill questionnaire in patients with chronic low back pain]. Qom Univ. Med. Sci. J. 2012; 6(1): 31-34.

24. Mendelson G, Selwood TS. Measurement of chronic pain: A correlation study of verbal and nonverbal scales. $J$ Psychopathol Behav Assess. 1981 Dec; 3(4): 263-269.

25. Vickers ER, Cousins MJ, Woodhouse A. Pain description and severity of chronic orofacial pain conditions. Aust Dent J. 1998 Dec; 43(6): 403-409.

26. Wasserstein D, Huston L, Nwosu S, et al. KOOS pain as a marker for significant knee pain two and six years after primary ACL reconstruction: a Multicenter Orthopaedic Outcomes Network (MOON) prospective longitudinal cohort study. Osteoarthr. Cartil. 2015;23(10):1674-1684.

27. Lepley AS, Pietrosimone B, Cormier ML. Quadriceps function, knee pain, and self-reported outcomes in patients with anterior cruciate ligament reconstruction. J. Athl. Train. 2018;53(4):337-346.

28. Tichonova A, Rimdeikienė I, Petruševičienė D, Lendraitienė E. The relationship between pain catastrophizing, kinesiophobia and subjective knee function during rehabilitation following anterior cruciate ligament reconstruction and meniscectomy: A pilot study. Medicina. 2016;52(4):229-237.

29. Culvenor AG, Øiestad BE, Holm I, et al. Anterior knee pain following anterior cruciate ligament reconstruction does not increase the risk of patellofemoral osteoarthritis at 15-and 20-year follow-ups. Osteoarthr. Cartil. 2017;25(1):30-33.

30. Kawaguchi K, Kuribayashi S, Nakayama S, Nakazato K, Fukubayashi T, Okinaga S. Lateral knee pain after outside-in anatomic double-bundle anterior cruciate ligament reconstruction using the TightRope RT. Knee Surg Relat Res. 2016 Mar;28(1):83.

31. Okoroha KR, Keller RA, Jung EK, et al. (2016) Pain assessment after anterior cruciate ligament reconstruction: bone-patellar tendon-bone versus hamstring tendon autograft. Orthop. J. Sports Med, 4(12):2325967116674924.

32. Pinczewski LA, Lyman J, Salmon LJ, et al. (2007) A 10-year comparison of anterior cruciate ligament reconstructions with hamstring tendon and patellar tendon autograft: acontrolled, prospective trial. Am J Sports Med, 35(4):564-574.

\section{Figures}




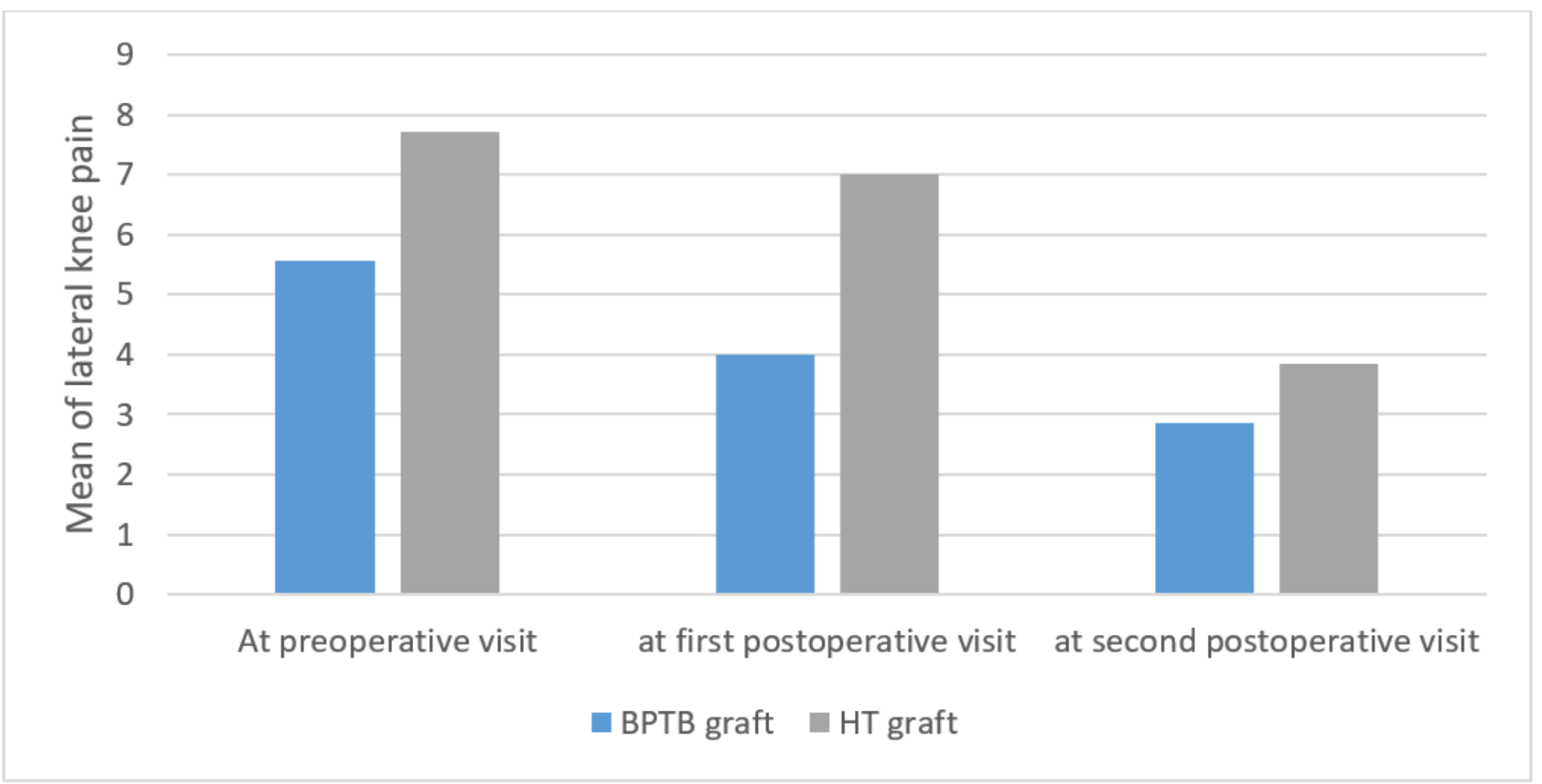

Figure 1

Mean lateral knee pain before and after ACL reconstruction according to usage of either BPTB or HT 